\title{
CARACTERÍSTICAS DE LA CANAL DE CORDEROS MERINO PRECOZ, SUFFOLK Y MESTIZOS DETERMINADAS POR ULTRASONOGRAFÍA LUMBAR Y EVALUACION DE SUS CORRELACIONES
}

\section{CARCASS TRAITS OF MERINO PRECOZ, SUFFOLK AND CROSSBRED LAMBS DETERMINED BY LUMBAR ULTRASONOGRAPHY}

\author{
Giorgio Castellaro G. ${ }^{*}$, Ximena García F. ${ }^{1}$, Juan Carlos Magofke S. ${ }^{1}$, y Gerónimo Marín F. ${ }^{1}$ \\ ${ }^{1}$ Universidad de Chile, Facultad de Ciencias Agronómicas, Dpto. Producción Animal, Santa Rosa \\ 11315, La Pintana, Santiago, Chile. \\ * Autor para correspondencia E-mail: gicastel@uchile.cl
}

\section{RESUMEN}

Se realizó un estudio con el propósito de comparar las características de canal en animales vivos de un grupo de corderos de tres genotipos (Merino precoz, Suffolk y mestizos Suffolk x Merino precoz). Se realizaron dos mediciones ecográficas en la zona lumbar entre la $12^{a}$ y $13^{a}$ costilla, separadas con un intervalo de 15 días, determinando la profundidad de lomo (PL), ancho del lomo (AL) y área del lomo (AOL) del músculo Longissimus dorsi, y el espesor de grasa dorsal (EGD) a dicho nivel. Las variables fueron analizadas mediante análisis de varianza, considerando como fuentes de variación el genotipo de los corderos, la fecha de evaluación y el efecto de la edad y peso de los mismos, variables que se ingresaron al modelo como covariantes. Adicionalmente, se determinaron correlaciones simples entre ellas. Las mediciones de AOL, PL, AL y EGD, a edad constante ( 140 días) y peso constante $(38,9 \mathrm{~kg})$ no fueron afectadas por el genotipo del cordero $(P>0,05)$, sugiriendo que las características de sus canales serían similares. Sin embargo, los corderos Merino precoz mostraron una tendencia a tener un mayor EGD en la primera medición. La variable PL tuvo alta y positiva correlación con AL y AOL y también con el EGD, lo que permite que dicha medición sea utilizada para estimar el desarrollo muscular. No obstante, PL debiera considerarse conjuntamente con EGD en los índices que evalúan la calidad de las canales de corderos in vivo, para evitar una excesiva adiposidad.

Palabras clave: calidad de canal, corderos Suffolk y Merino precoz, cruzamiento terminal, ultrasonografía.

\section{ABSTRACT}

A study was conducted to compare carcass traits in live animals in a group of lambs of three different genotypes (Merino precoz, Suffolk and crossbreds Suffolk x Merino precoz). Two ultrasonographic measurements were made with an interval of 15 days in the lumbar zone between the $12^{\text {th }}$ and $13^{\text {th }}$ rib, determining depth (PL, cm), width $(\mathrm{AL}, \mathrm{cm})$ and area $\left(\mathrm{AOL}, \mathrm{cm}^{2}\right)$ of the Longissimus dorsi muscle and backfat thickness (EGD, cm) at that level. Variables were analyzed using analysis of variance, considering lamb's genotype, date of assessment and the effect of age and weight as sources of variation, which were entered into the model as covariates. Additionally, simple correlations were determined between them. Values for AOL, PL, AL and EGD at constant age (140 days) and constant weight $(38.9 \mathrm{~kg})$ were not affected by lamb's genotype $(P>0.05)$, suggesting that the characteristics of their carcasses would be similar. Nevertheless, Merino precoz lambs tended to have a greater EGD in the first measurement. PL was highly and positively correlated with AL and AOL, as well as with 
EGD so that this measurement can be used to estimate muscle development. However, PL should be considered in conjunction with EGD in the indices that evaluate in vivo lamb carcass quality in order to prevent excessive adiposity of this.

Key words: carcass quality, Suffolk and Merino precoz lambs, terminal crossbreeding, ultrasonography.

\section{INTRODUCCION}

En los últimos años se han desarrollado investigaciones para buscar métodos que evalúen de forma objetiva la composición y calidad de canales de corderos de carne (Junkuszew y Ringdorfer, 2005; Teixeira et al., 2006). Estos métodos tienen como propósito lograr evaluaciones más precisas que la apreciación visual, y que muestren una buena repetibilidad, mejorando el proceso de selección de carneros utilizados, para contribuir al aumento de la productividad y de los ingresos de la explotación ganadera. Entre estas se puede citar a aquellas que se basan en imágenes, como tomografía axial computarizada y el ultrasonido en tiempo real, las que tienen la gran ventaja de no ser invasivas o destructivas (Teixeira et al.; 2006; Grill et al., 2015).

La aplicación de la ultrasonografía en producción animal es una herramienta de uso común desde varios años, y ha sido utilizada para medir el espesor de grasa dorsal, de la pared del cuerpo, y dimensiones del músculo Longissimus dorsi, para estimar la calidad de las canal en el animal en vivo (Bianchi, 2006; Parraguez, 2007; Grill et al., 2015). Basándose en los valores de estas mediciones, se han propuesto criterios para realizar una selección temprana de los reproductores (Bianchi et al., 2006).

El objetivo del ensayo fue evaluar, comparar y correlacionar algunas características de canal, determinadas mediante ecografía lumbar, en corderos de las razas Merino (M), Suffolk (S), y mestizos Merino $\times$ Suffolk (SM), productos de un sistema de cruzamiento terminal.

\section{MATERIALES Y MÉTODOS}

El estudio se realizó en la Estación Experimen-

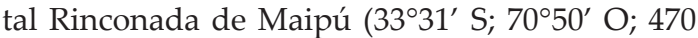
m.s.n.m.), perteneciente a la Facultad de Ciencias Agronómicas de la Universidad de Chile, durante el año 2007. Se evaluaron corderos machos de los genotipos Merino precoz (MP), Suffolk Down (S) y corderos mestizos provenientes de un cruzamiento terminal entre hembras Merino precoz y carneros Suffolk (SM), de peso y edad similar (37,4 kg, 132 días). Se utilizaron 12 corderos por cada genotipo, número que fue determinado en función de la variabilidad observada en estas variables en experimentos similares (Bianchi, 2006).
Los corderos permanecieron junto a sus madres desde su nacimiento hasta el destete, teniendo como única fuente de alimentación un pastizal natural anual de clima mediterráneo. Posterior al destete, fueron mantenidos en el mismo pastizal. En los corderos se midió el peso vivo (PV) y la condición corporal (escala 1-5), y se estimaron la profundidad del lomo (PL), ancho del lomo (AL) y área del L. dorsi (AOL) y el espesor de grasa dorsal (EGD) a nivel de la $12^{\mathrm{a}}$ costilla, mediante ecografía lumbar. Las ecografías se realizaron utilizando un ecógrafo (Esaote Pie Medical, modelo Aquila Vet, con un transductor lineal de $6 \mathrm{MHz}$, Maastrich, The Netherlands). Para mejorar la transducción de las ondas de sonido, se utilizó un gel hidrosoluble sobre el transductor para suprimir el aire entre este y la piel del animal. Complementariamente y en la misma zona de medición, se aplicó aceite vegetal sobre la piel para desplazar el aire presente en la lana donde el gel no era capaz de penetrar. Se efectuaron dos campañas de mediciones, separadas por 15 días. Los datos fueron analizados utilizando un diseño completamente al azar, mediante un modelo estadístico que contempló el efecto de la fecha de medición y del genotipo del cordero como principales fuentes de variación. El modelo también incluyó un covariante por peso o edad del cordero, debido a posibles variaciones entre los animales que pudiesen ser atribuidos a estos factores:

$$
Y_{i j_{k}}=\mu+G_{i}+F M_{j}+\beta \cdot x_{k}+\varepsilon_{i j k}
$$

donde $\mathrm{Y}_{\mathrm{ijk}}$ es la variable respuesta (profundidad, ancho, largo del L. dorsi y espesor de grasa dorsal) del k ${ }^{\text {esimo }}$ cordero; $\mu$ es el promedio general; $G_{i}$ es el efecto del ísimo genotipo ( $i=$ Suffolk; Merino precoz y Suffolk x Merino precoz); FM es el efecto de la jesima fecha de medición; $\beta \cdot x_{k}$ es la variable covariante por peso o por edad del cordero, y $\varepsilon_{\mathrm{ijk}}$ representa al error experimental.

El análisis de variancia realizado en el ensayo fue del tipo III, utilizando un intervalo de confianza del 95\%. Para detectar diferencias estadísticas entre los tratamientos, se aplicó la prueba de comparación múltiple Student-Newman-Keuls (SNK), con una confianza del 95\%. Complementariamente, se determinó una matriz de correlaciones de Pearson entre todas las variables evaluadas, para determinar el grado de asociación entre ellas. Todos los análisis anteriores fueron 
realizados utilizando un software estadístico especializado (SAS, 1996).

\section{RESULTADOS Y DISCUSIÓN}

Los resultados obtenidos en el análisis de varianza, mostraron que las variables covariantes, edad o peso, no tuvieron efectos significativos ( $P$ $>0,05)$, lo que indica que los animales utilizados en este ensayo fueron relativamente homogéneos en cuanto a estas variables (140 días y $38,9 \mathrm{~kg}$, en edad y peso promedio, respectivamente) y que estas no afectaron las mediciones de AOL, EGD, PL y AL. El único factor que resultó ser significativo $(\mathrm{P} \leq 0,05)$ fue la fecha de medición, hecho esperable debido a que como consecuencia del crecimiento y desarrollo de los animales, se producen modificaciones en el peso y en la composición corporal (Bianchi et al., 2006; Pérez et al., 2007). El genotipo no afectó significativamente las mediciones y no tuvo interacción significativa $(\mathrm{P}$ $>0,05)$ con la fecha de medición.

\section{Espesor de grasa dorsal (EGD)}

Los resultados obtenidos en las ecografías lumbares muestran que el genotipo no afectó de manera significativa $(P>0,05)$ el espesor de grasa dorsal (EGD), tanto en la primera (132 \pm 7 días de edad) como en la segunda fecha de medición (147 \pm 7 días de edad) de medición (Tabla $1)$, no obstante, se observó una tendencia a un mayor EGD en corderos Merino Precoz. Es de interés visualizar el incremento que se produjo en el EGD (a edad promedio) entre las dos fechas de medición, el cual fue de un 23,9; 27,2; y $50,4 \%$ en Merino precoz, mestizos y Suffolk, respectivamente, lo que estaría indicando que esta última raza es un genotipo que presenta una tasa de deposición adiposa más tardía y de mayor magnitud en comparación a los corderos Merino precoz. Una tendencia similar se observó cuando la comparación se realizó a peso constante $(38,9 \mathrm{~kg})$, no obstante en este caso, los porcentajes de incremento del espesor graso fueron menores (Tabla 1). A pesar de que el peso vivo de los corderos fue relativamente alto al momento de efectuar las mediciones, los espesores de grasa dorsal determinados en este estudio no fueron excesivos y son levemente inferiores a los reportados por la literatura para corderos Merinos puros y en cruzas con razas de carne de cuatro meses de edad (Bianchi, 2006).

Tabla 1. Espesor de grasa dorsal, área, profundidad y ancho del Longissimus dorsi, en corderos de diferentes genotipos.

Table 1. Backfat thickness and area, depth and width of the Longissimus dorsi muscle in lambs of different genotypes.

\begin{tabular}{|c|c|c|c|c|c|c|}
\hline & \multicolumn{3}{|c|}{ A edad promedio (140 días) } & \multicolumn{3}{|c|}{ A peso promedio $(38,9 \mathrm{~kg})$} \\
\hline Genotipo & $\begin{array}{l}\text { Primera } \\
\text { fecha }\end{array}$ & Segunda fecha & $\begin{array}{c}\text { Incremento } \\
\%\end{array}$ & $\begin{array}{c}\text { Primera } \\
\text { fecha }\end{array}$ & $\begin{array}{l}\text { Segunda } \\
\text { fecha }\end{array}$ & $\begin{array}{c}\text { Incremento } \\
\%\end{array}$ \\
\hline \multicolumn{7}{|c|}{ Espesor de grasa dorsal (EGD, mm) } \\
\hline Merino & $1,63 \mathrm{aB}$ & $2,02 \mathrm{aA}$ & 23,9 & $1,67 \mathrm{aB}$ & $1,93 \mathrm{aA}$ & 15,6 \\
\hline Mestizo & $1,51 \mathrm{aB}$ & $1,92 \mathrm{aA}$ & 27,2 & $1,61 \mathrm{aB}$ & $1,90 \mathrm{aA}$ & 18,0 \\
\hline Suffolk & $1,31 \mathrm{aB}$ & $1,97 \mathrm{aA}$ & 50,4 & $1,46 \mathrm{aB}$ & $1,93 \mathrm{aA}$ & 32,2 \\
\hline \multicolumn{7}{|c|}{ Área del ojo del lomo (AOL, $\left.\mathrm{cm}^{2}\right)$} \\
\hline Merino & $11,72 \mathrm{aB}$ & $14,39 \mathrm{aA}$ & 22,8 & $11,86 \mathrm{aB}$ & $14,06 \mathrm{aA}$ & 18,5 \\
\hline Mestizo & $11,60 \mathrm{aB}$ & 14,24 aA & 22,8 & $11,90 \mathrm{aB}$ & 14,08 aA & 18,3 \\
\hline Suffolk & $11,13 \mathrm{aB}$ & $13,89 \mathrm{aA}$ & 24,8 & $11,65 \mathrm{aB}$ & $13,77 \mathrm{aA}$ & 18,2 \\
\hline \multicolumn{7}{|c|}{ Profundidad del lomo (PL, cm) } \\
\hline Merino & $2,26 \mathrm{aB}$ & $2,48 \mathrm{aA}$ & 9,7 & $2,26 \mathrm{aB}$ & $2,48 \mathrm{aA}$ & 9,7 \\
\hline Mestizo & $2,25 \mathrm{aB}$ & $2,49 \mathrm{aA}$ & 10,7 & $2,26 \mathrm{aB}$ & $2,51 \mathrm{aA}$ & 11,1 \\
\hline Suffolk & $2,21 \mathrm{aB}$ & $2,50 \mathrm{aA}$ & 13,1 & $2,24 \mathrm{aB}$ & $2,48 \mathrm{aA}$ & 10,7 \\
\hline \multicolumn{7}{|c|}{ Ancho del lomo (AL, cm) } \\
\hline Merino & $6,72 \mathrm{aB}$ & 7,39 aA & 9,8 & $6,73 \mathrm{aB}$ & 7,37 aA & 9,5 \\
\hline Mestizo & $6,63 \mathrm{aB}$ & $7,29 \mathrm{aA}$ & 10,0 & $6,65 \mathrm{aB}$ & $7,27 \mathrm{aA}$ & 9,3 \\
\hline Suffolk & $6,74 \mathrm{aB}$ & 7,29 aA & 8,2 & $6,77 \mathrm{aB}$ & 7,28 aA & 7,5 \\
\hline
\end{tabular}

Letras minúsculas diferentes en la columna indican diferencias significativas $(P \leq 0,05)$. Letras mayúsculas diferentes en las filas covariante indican diferencias significativas $(\mathrm{P} \leq 0,05)$. 


\section{Área muscular del lomo (AOL)}

En relación con el área del ojo del lomo, no se observaron diferencias significativas entre los tres genotipos, tanto para la primera como para la segunda fecha de medición $(P>0,05)$. Los incrementos del AOL fueron mucho más parejos que en el caso anterior (Tabla 1). Los valores calculados a edad promedio (140 días), muestran que los corderos Suffolk presentaron el incremento más alto, con un $24,8 \% \mathrm{v} / \mathrm{s} 22,8 \%$ en relación a sus contemporáneos Merino precoces y mestizos (Tabla 1). Cuando los datos fueron analizados a peso promedio $(38,9 \mathrm{~kg})$, los incrementos fueron del orden del $18 \%$ en los tres genotipos. Valores muy similares a los de este estudio fueron encontrados en corderos Merino puros y cruzas Merinos con genotipos carniceros de cuatro meses de edad (Bianchi, 2006).

\section{Profundidad de lomo (PL)}

Al igual que en los dos casos anteriores, las mediciones de profundidad del $L$. dorsi no presentaron diferencias significativas $(P>0,05)$ entre genotipos en ninguna de las dos fechas de medición. A diferencias de los casos anteriores, el incremento en esta variable fue bajo y no superó el $13,1 \%$ tanto para el análisis a edad y peso constante (Tabla 1). Al igual que lo acontecido con el EGD, los corderos de genotipo Suffolk presentaron los mayores incrementos.

\section{Ancho del lomo (AL)}

Es relevante comentar que los datos obtenidos en las ecografías del ancho del lomo no presentaron un comportamiento con una distribución normal. Debido a esta situación, previo a efectuar el análisis estadístico se realizó una corrección de los datos mediante el método Boxcox para otorgar normalidad a la información. El comportamiento del ancho de lomo no difirió de las otras variables evaluadas, no encontrándose diferencias significativas $(P>0,05)$ entre los genotipos tanto para la primera como la segunda fecha de medición. En esta variable los porcentajes de incrementos entre ambas fechas fueron menores, no superando el 10\% (Tabla 1).

Aun cuando los resultados obtenidos en las mediciones de EGD y dimensiones del $L$. dorsi (Tabla 1) no mostraron diferencias significativas entre genotipos $(P>0,05)$, se debe descartar que el método de medición utilizado (ultrasonografia) sea poco preciso. Son numeroso los autores (Cadavez et al., 2000a; Cadavez et al., 2000b; Junkuszew y Ringdorfer, 2005; Bianchi, 2006; Teixeira et al., 2006; Thériault et al., 2009; Maximini et al., 2012; Grill et al., 2015) que han estudiado el uso de la ultrasonografía en producción animal, para evaluar características de las cana- les. Muchos de los trabajos anteriores han considerado la evaluación de este método mediante el cálculo de la correlación entre las mediciones efectuadas in vivo y las mismas post morten, especialmente en variables relacionadas con las dimensiones del L. dorsi y espesor graso. Estas correlaciones presentan altos valores, demostrando la alta precisión de este método (Thériault et al., 2009; Grill et al., 2015).

A pesar que los resultados de este estudios no mostraron diferencias significativas respecto a las dimensiones del L. dorsi y el espesor de grasa dorsal, la literatura muestra que, al menos a peso constante, los corderos mestizos producto de cruzamientos de razas maternas con carneros de aptitud carnicera, exhiben mayor desarrollo del músculo $L$. dorsi y mayor proporción de carne, sin verse afectado el porcentaje de grasa, en comparación a los corderos puros de la raza materna (Bianchi, 2006). No obstante lo anterior, el hecho de no haber encontrado diferencias a favor de los corderos SMP en las mediciones asociadas a la canal, podría explicarse por el bajo nivel de heterosis individual que presentan dichas características, impidiendo registrar diferencias (Bradford, 2002). Un comportamiento similar fue reportado por Macías-Cruz et al. (2010) en corderos Pelibuey puros y mestizos F1 con razas Dorper y Katahdin.

La matriz de correlaciones entre las variables evaluadas, considerando el conjunto de datos recolectados en ambas fechas de medición, se presenta en la Tabla 2. Los resultados muestran la inexistencia de una correlación significativa entre el peso vivo y la condición corporal, así como entre el peso vivo y la profundidad del lomo ( $\mathrm{P}>$ $0,05)$. Estos resultados difieren a los encontrados por Vargas et al. (2008), los cuales determinaron una correlación positiva entre el peso vivo y la condición corporal del orden del 35 al 37\%, medida en ovinos de pelo de 5 a 8 meses de edad. Con respecto a los valores de las correlaciones entre las otras variables, todas ellas resultaron ser altamente significativas ( $\mathrm{P} \leq 0,001)$.

Los corderos de mayor peso vivo tendieron a ser también los que presentaron mayor área del L. dorsi y espesor graso, donde la correlación fue de 41 y $36 \%$, respectivamente.

Los resultados obtenidos con respecto a la correlación entre la CC y EGD fue 37\%, lo que era esperable ya que la CC es una medida del grado de engrasamiento del animal (SCA, 2007). Bianchi (2006) cita valores aún más altos, del orden del $51 \%$.

Las correlaciones más altas fueron las que relacionaron las dimensiones del $L$. dorsi, en especial la existente entre el área del ojo del lomo con su ancho y profundidad (Tabla 2). Con res- 
Tabla 2. Coeficientes de correlación de Pearson (r) entre el peso vivo (PV), condición corporal (CC), espesor de grasa dorsal (EGD), área (AOL), profundidad (PL) y ancho (AL) del músculo Longisimus dorsi, medidos en corderos.

Table 2. Pearson correlation coefficients ( $r$ ) between liveweight (PV), body condition (CC), backfat thickness (EGD), area (AOL), depth (PL) and width (AL) of the Longisimus dorsi muscle measured in lambs.

\begin{tabular}{lllllll}
\hline & CC & PV & EGD & AOL & PL & AL \\
\hline CC, escala 1-5 & ---- & & & & & \\
PV, kg & $0,12 \mathrm{~ns}$ & ---- & & & & \\
EGD, mm & $0,37^{* *}$ & $0,36^{* *}$ & ---- & & & \\
AOL, cm ${ }^{2}$ & $0,31^{* *}$ & $0,41^{* *}$ & $0,56^{* *}$ & ---- & & \\
PL, cm & $0,47^{* *}$ & $0,12 \mathrm{~ns}$ & $0,51^{* *}$ & $0,71^{* *}$ & --- & \\
AL, cm & $0,35^{* *}$ & $0,53^{* *}$ & $0,57^{* *}$ & $0,78^{* *}$ & $0,52^{* *}$ & --- \\
\hline
\end{tabular}

ns: no significativo

**: $\mathrm{P} \leq 0,001$

pecto a esta última, es posible destacar la gran utilidad que ella presenta, ya que puede ser utilizada como una medida indirecta del área del $L$. dorsi, debido a su mayor facilidad de medición. $\mathrm{Al}$ respecto, en la Fig. 1 se presenta la ecuación de regresión obtenida entre ambas variables, la que resultó ser altamente significativa $(\mathrm{P}<0,01)$, con un $\mathrm{R}^{2}$ de $49,85 \%$ e indica que por cada centímetro de incremento en la profundidad del $L$. dorsi se produce una variación de $5,66 \mathrm{~cm}^{2}$ en el área de este músculo.

También fueron destacables las correlaciones existente entre las dimensiones del L. dorsi con el espesor de grasa dorsal. El grado de correlación obtenido (56\%) corrobora la tendencia que indica que aquellos animales que tienen un mayor desarrollo del músculo L. dorsi, son también los que tienen mayor espesor de grasa dorsal, aspecto que también fue determinado en el estudio de Grill et al. (2015), quienes mencionan correlaciones entre 81 y $88 \%$ entre estas mediciones. En la Fig. 2 se presenta la regresión lineal obtenida entre estas variables, la que fue altamente significativa $(P<0,01)$, pero con un $R^{2}$ más bajo $(31,16 \%)$, al obtenido entre la profundidad y el área muscular. En este caso, por cada centímetro cuadrado de incremento en el área del L. dorsi se produce una variación de $0,136 \mathrm{~mm}$ en el espesor graso.

Al utilizar como único criterio de selección el área del L. dorsi (o su profundidad), se estará también seleccionando indirectamente a aquellos animales que presentan mayor espesor graso, lo que a largo plazo puede llevar a favorecer a aquellos corderos de mayor desarrollo muscular pero también con mayor grado de engrasamiento de sus canales. Esta afirmación se basa en el hecho de que la correlación genética entre estas características es moderadamente positiva, siendo su valor del orden de 33\% (Simm, 1998; Jones et al., 2004; Maximini et al., 2012).

Seleccionar reproductores utilizando solamente como criterio el desarrollo muscular (medido a través del área o la profundidad del $L$. dorsi) puede ser riesgoso, ya que ello producirá en el largo plazo animales con canales sobre-engrasadas. Simm (1998) propuso integrar dichas mediciones en un índice de selección que considera el peso vivo y la profundidad del $L$. dorsi con una ponderación positiva y el EGD con ponderación negativa, lo que también es sugerido en los índices de selección propuestos por Márquez et al. (2013) para carneros terminales.

\section{CONCLUSIONES}

Los corderos de los genotipos analizados, con edad y peso promedio de 140 días y $38,9 \mathrm{~kg}$, respectivamente, no presentaron diferencias significativas en las mediciones ecográficas lumbares, lo que estaría indicando que las características de sus canales serían similares. La profundidad del L. dorsi (PL) tiene alta y positiva correlación con el ancho (AL) y el área del ojo del lomo (AOL), por lo cual puede ser utilizada como un buen predictor del tamaño de este músculo en corderos en pie. Dado la alta correlación existente entre PL y AOL con el espesor de grasa dorsal (EGD), esta última medición debiera considerarse conjuntamente con los predictores del tamaño muscular en los índices que evalúan in vivo la calidad de las canales de corderos, para evitar seleccionar corderos de alto desarrollo muscular pero con canales con excesiva grasa. 


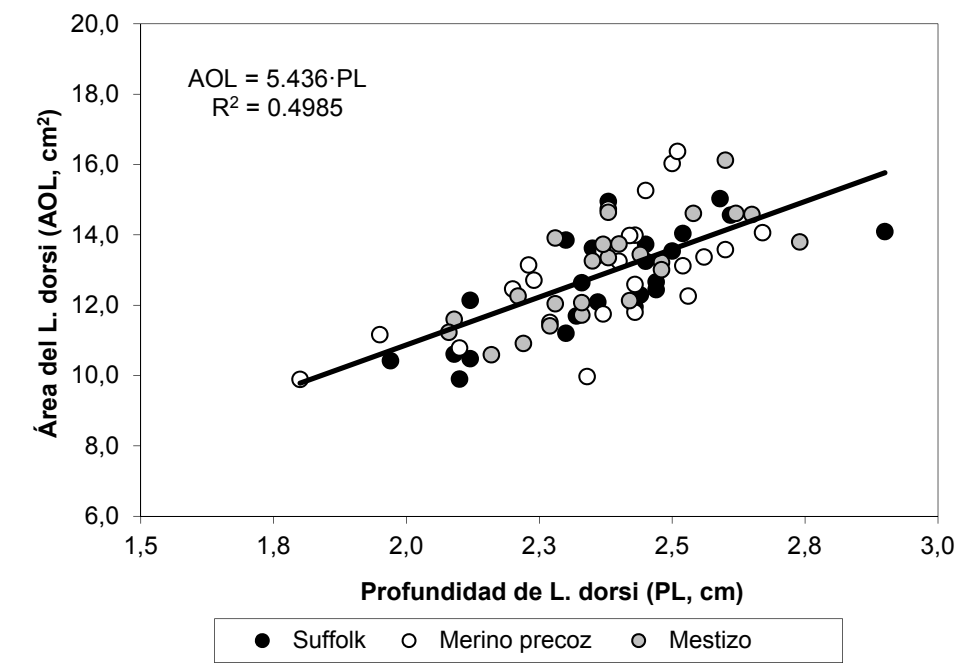

Fig. 1. Regresión lineal entre la profundidad del Longissimus dorsi (PL) y su área (AOL) para corderos Merinos precoz, Suffolk y mestizos.

Fig. 1. Linear regression between depth of the Longissimus dorsi muscle (PL, cm) and area (AOL, $\mathrm{cm}^{2}$ ) in Merino precoz, Suffolk and crossbred lambs.

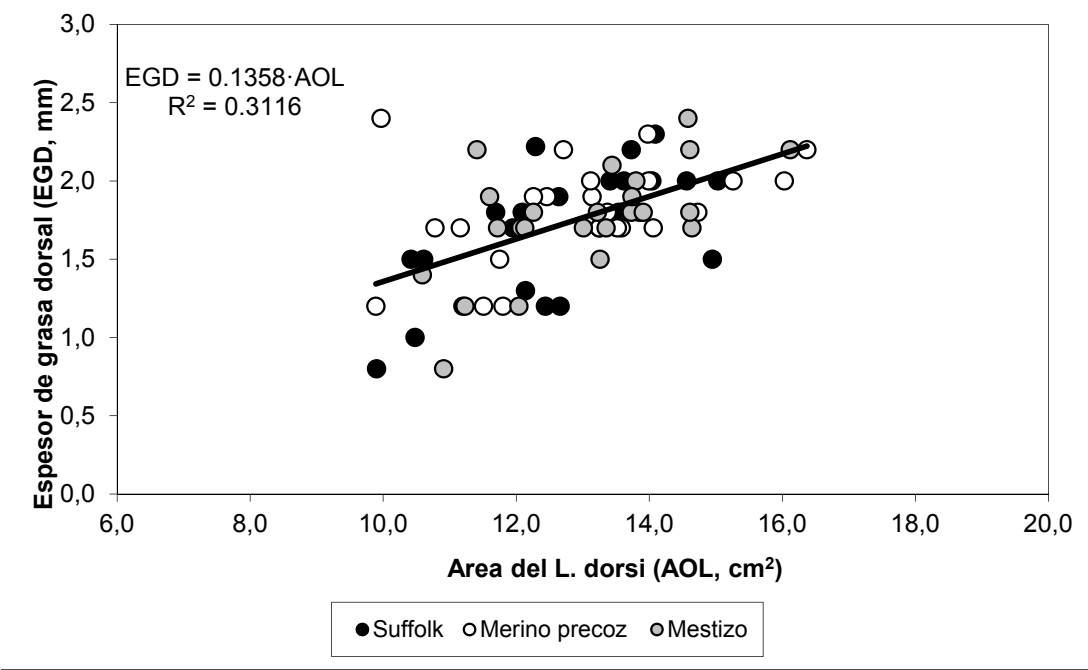

Fig. 2. Regresión lineal entre el área del músculo Longissimus dorsi y el espesor de grasa dorsal, en corderos Merinos precoz, Suffolk y mestizos.

Fig. 2. Linear regression between area of the Longissimus dorsi muscle and backfat thickness in Merino precoz, Suffolk and crossbred lambs.

\section{LITERATURA CITADA}

Bianchi, G. 2006. Alternativas tecnológicas para la producción de carne ovina de calidad en sistemas pastoriles. 283 p. Editorial Hemisferio Sur, Montevideo, Uruguay.

Bianchi, G., G. Garibotto, O. Feed, O. Bentancur, y J. Franco. 2006. Efecto del peso al sacrificio sobre la calidad de la canal y de la carne de corderos Corriedale puros y cruza. Archivos Medicina Veterinaria 38(2):161-165

Bradford, E. 2002. Breeding and selection. p. 1-80. In Sheep Production Handbook. American Sheep Industry Association, Fort Collins, Colorado, USA. 
Cadavez, V.A.P., A. Teixeira, R. Delfa, y S. Rodríguez. 2000a. Utilización de ultrasonidos y el peso de la canal caliente para la predicción de la composición de la canal en corderos. Producción Ovina y Caprina SEOC XXV:169172.

Cadavez, V. A. P., A. Teixeira, R. Delfa, y S. Rodríguez. 2000b. Utilización de ultrasonidos y el peso vivo para la predicción in vivo de la composición de la canal en corderos. p. 165168. En Producción Ovina y Caprina SEOC $\mathrm{N}^{\circ}$ 25. XXV Jornadas Científicas y IV Internacionales. 28-30 septiembre 2000. SEOC, Teruel, España.

Grill, L., F. Ringdorfer, R. Baumung, and B. Fuerst-Waltl. 2015. Evaluation of ultrasound scanning to predict carcass composition of Austrian meat sheep. Small Ruminant Research 123:260-268

Jones, H.E., R.M. Lewis, M.J. Young, and G. Simm. 2004. Genetic parameters for carcass composition and muscularity in sheep measured by X-ray computer tomography, ultrasound and dissection. Livest. Prod. Sci. 90:167-179.

Junkuszew, A., and F. Ringdorfer. 2005. Computer tomography and ultrasound measurement as methods for the prediction of the body composition of lambs. Small Ruminant Research 56:121-125.

U. Macías-Cruz, F.D. Álvarez-Valenzuela, J. Rodríguez-García, A. Correa-Calderón, N.G. Torrentera-Olivera, L. Molina-Ramírez, y L. Avendaño-Reyes. 2010. Crecimiento y características de canal en corderos Pelibuey puros y cruzados F1 con razas Dorper y Katahdin en confinamiento. Arch. Med. Vet. 42:147-154.

Márquez, G. C., W. Haresign, M.H. Davies, R. Roehe, L. Bünger, G. Simm, and R.M. Lewis. 2013. Index selection in terminal sires improves lamb performance at finishing. Journal of Animal Science 91(1):38-43.

Maximini, L., D.J. Brown, R. Baumung, and B. Fuerst-Waltl. 2012. Genetic parameters of ultrasound and computer tomography scan traits in Austrian meat sheep. Livestock Science 146:168-174.
Parraguez, V. 2007. La ecografía como herramienta para la producción de pequeños rumiantes y camélidos sudamericanos. Vo Congreso de Especialistas en Pequeños Rumiantes y Camélidos Sudamericanos. 2, 3 y 4 de mayo de 2007. Mendoza, Argentina. Asociación Latinoamericana de Especialistas en Pequeños Rumiantes y Camélidos Sudamericanos (ALEPRYCS), Mendoza, Argentina.

Pérez, P., M. Maino, C. Köbrich, M. Morales, y J. Pokniak. 2007. Efecto del peso de sacrificio y sexo sobre la canal de corderos lactantes del cruce Suffolk Down x Merino Precoz alemán. Revista Científica FCV-LUZ 17(6):621-626.

SAS. 1996. Versión 6.12 ed.. SAS Institute Inc., Cary, North Caroline, USA.

SCA. 2007. Nutrient Requirement of Domesticated Ruminants. 296 p. Standing Committee on Agriculture (SCA), CSIRO Publications, Melbourne, Australia.

Simm, G. 1998. Genetic improvement of cattle and sheep. 433 p. Farming Press, Tonbridge, United Kingdom.

Teixeira, A., S. Matos, S. Rodrigues, R. Delfa, and V. Cadavez. 2006. In vivo estimation of lamb carcass composition by real-time ultrasonography. Meat Science 74:289-295.

Thériault, M., C. Pomar, F.W. Castonguay. 2009. Accuracy of real-time ultrasound measurements of total tissue, fat, and muscle at different measuring sites in lamb. Journal of Animal Science 87:1801-1813.

Vargas, F., I. Vergara, M.A. Pérez, y J. De Lucas. 2008. Características de la canal de ovinos de pelo mediante uso de ultrasonografia y evaluación post morten. p. 465-468. XXXIII Jornadas Científicas y XII Jornada Internacional de Ovinotecnia y Caprinotecnia. SEOC. 24-27 de septiembre de 2008. Junta de Andalucía, Consejería de Agricultura y Pesca, Dirección General de Planificación y Análisis de Mercados, Servicio de Publicaciones y Divulgación, Almería, España. 